\title{
Color Processing in Macaque Striate Cortex: Relationships to Ocular Dominance, Cytochrome Oxidase, and Orientation
}

\author{
CAROLE E. LANDISMAN AND DANIEL Y. TS'O \\ The Rockefeller University, New York, New York 10021; and Baylor College of Medicine, Houston, Texas 77030
}

Received 17 November 1999; accepted in final form 8 February 2002

Landisman, Carole E. and Daniel Y. Ts'o. Color processing in macaque striate cortex: relationships to ocular dominance, cytochrome oxidase, and orientation. J Neurophysiol 87: 3126-3137, 2002; 10.1152/jn.09569.1999. We located clusters of color-selective neurons in macaque striate cortex, as mapped with optical imaging and confirmed with electrophysiological recordings. By comparing responses to an equiluminant red/green stimulus versus a high-contrast luminance stimulus, we were able to reveal a patchy distribution of color selectivity. Other color imaging protocols, when compared with electrophysiological data, did not reliably indicate the location of functional structures. The imaged color patches were compared with other known functional subdivisions of striate cortex. There was a high degree of overlap of the color patches with the cytochromeoxidase (CO) blobs. The patches were often larger than a single blob in size, however, and in some instances spanned two neighboring blobs. More than one-half (56\%) of the color-selective patches seen in optical imaging were not confined to one ocular dominance (OD) column. Almost one-quarter of color patches (23\%) extended across OD columns to encompass two blobs of different eye preference. We also compared optical images of orientation selectivity to maps of color selectivity. Results indicate that the layout of orientation and color selectivity are not directly related. Specifically, despite having similar scales and distributions, the maps of orientation and color selectivity were not in consistent alignment or registration. Further, we find that the maps of color selectivity and of orientation are each only loosely related to maps of OD. This description stands in contrast to a common depiction of color-selective regions as identical to $\mathrm{CO}$ blobs, appearing as pegs in the centers of OD columns in the classical "ice cube" model. These results concerning the pattern of color selectivity in V1 support the view (put forth in previous imaging studies of the organization of orientation and ocular dominance) that there is not a fundamental registration of functional hypercolumns in V1.

\section{N T R O D U C T I O N}

Our understanding of the organization of primate striate cortex (V1) has been enhanced greatly by the discovery of cytochrome oxidase-rich blobs (Wong-Riley 1979). The blobs appear as a regular pattern of patches seen in tangential sections through layers I, II/III, IVB, V, and VI, when stained for cytochrome-oxidase (CO) activity (Horton 1984; Horton and Hubel 1981; Wong-Riley 1979). Because CO is a marker for metabolic activity and its level of activity might therefore reflect underlying neuronal properties, several studies have examined the relationship between $\mathrm{CO}$ blobs and other aspects

Present address and address for reprint requests: C. E. Landisman, Dept. of Neuroscience, Box 1953, Brown University, Providence, RI 02912 (E-mail: Carole_Landisman@Brown.edu). of the functional architecture of V1. For example, Horton and Hubel (1981) noted that the CO blobs lie along the centers of ocular dominance (OD) columns.

\section{Functional layout and role of the CO blobs}

Electrophysiological studies of the functional properties of $\mathrm{CO}$ blobs found that they contain neurons having monocular, color-selective, and unoriented response properties (Livingstone and Hubel 1984; Ts'o and Gilbert 1988). These findings have been supported by other techniques, such as 2-deoxyglucose uptake (2-DG) and optical imaging, that provide a more macroscopic view of the spatial relationship of functional properties to CO blobs (Blasdel 1992a,b; Tootell et al. 1988; Ts'o et al. 1990). Horton (1984) used 2-DG to demonstrate that cells in the blobs respond well to monocular stimulation with achromatic gratings at all orientations. In an optical imaging study, Ts'o et al. (1990) found that when achromatic sinewave gratings were used to visualize OD columns, the regions that showed the strongest signal correspond to CO blobs. Based on these findings, along with the electrophysiological studies, it was concluded that the blobs represent monocular sites of color processing in primate striate cortex.

The role of the blobs in color vision, however, has been called into question by several lines of evidence. $\mathrm{CO}$ blobs of similar size and distribution to those found in the macaque are also found in primates lacking well-developed color vision (Jacobs 1977) such as owl monkeys (Horton 1984) and galagos (Horton 1984; Lachica and Cassagrande 1992). Thus it was concluded that $\mathrm{CO}$ blobs are involved in functions other than color processing. Moreover, the original electrophysiological studies of blob cells reported a significant population of unoriented but non-color-selective (broadband) cells in the blobs (Livingstone and Hubel 1984; Ts'o and Gilbert 1988). Furthermore, Lennie et al. (1990) reported no clear concentration of color-selective cells in the blob regions, and Leventhal et al. (1995) found no correlation between the CO staining pattern in $\mathrm{V} 1$ and the color and orientation response properties of individual neurons. Electrode placements in the latter two studies, however, were not guided by prior knowledge of the location of the blobs. Thus these studies did not examine in detail, with multiple penetrations, the receptive field properties of cells within known blobs.

\footnotetext{
The costs of publication of this article were defrayed in part by the payment of page charges. The article must therefore be hereby marked "advertisement" in accordance with 18 U.S.C. Section 1734 solely to indicate this fact.
} 
Other functional properties ascribed to $\mathrm{CO}$ blobs include preferences for low spatial frequency stimulation. Silverman et al. (1989) reported an organization for spatial frequency tuning in V1, with a preference for low spatial frequencies found among neurons within the blobs and a radial increase in preferred spatial frequency with increasing distance from blob centers (also see Edwards et al. 1995).

In the present study, we used optical imaging to determine whether there is any functional segregation in macaque V1 for cells exhibiting properties such as those originally reported for neurons in the blobs including monocularity, color opponency, and poor orientation tuning. We first created an imaging protocol to successfully show the location of color-selective cells. After confirming the reliability of this mapping protocol with electrophysiology, we used these color maps to compare the layout and distribution of color-selective regions in V1 to other functional maps and to the CO blobs. Our data indicate that, despite the high degree of organization of functional maps, a lattice-like relationship of these functional maps does not exist.

\section{METHODS}

Eighteen Macaca fascicularis monkeys were used in the experiments describe here and in related experiments, results to be published separately. The animals, weighing $3.15-6.35 \mathrm{~kg}$, were initially given ketamine $\mathrm{HCl}(20 \mathrm{mg} / \mathrm{kg} \mathrm{im})$, then anesthetized by sodium thiopental $\left(20 \mathrm{mg} / \mathrm{kg}\right.$ iv) and maintained on sodium thiopental $\left(1-2 \mathrm{mg} \cdot \mathrm{kg}^{-1}\right.$. $\mathrm{h}^{-1}$ iv) for the rest of the experiment. Electrocardiogram, temperature, and electroencephalogram were monitored. A craniotomy approximately one square centimeter in size was made over parafoveal V1 (approximately $2-4^{\circ}$ ). A stainless steel optical chamber was secured with dental acrylic over the hole. All surgery was performed under sterile conditions. The animal was then paralyzed with vecuronium bromide $\left(0.1 \mathrm{mg}^{-1} \cdot \mathrm{kg} \cdot \mathrm{h}^{-1}\right.$ iv $)$ and artificially respired at a rate to maintain expired $\mathrm{CO}_{2}$ at $4-4.5 \%$. The eyes were refracted with a retinoscope and fitted with appropriate contact lenses to focus on the stimulus monitor. Foveae were located with a fundus camera and back projected onto the tangent stimulus monitor.

Prior to exposing the entire square centimeter of cortical surface, a small hole was made in the dura for preliminary electrode recording to determine the location of receptive fields with respect to the stimulation monitor (which could be moved to align with the receptive fields). When necessary, a prism was used in front of one eye to modify the location of fixation so that receptive fields for both eyes were well positioned on the monitor. After this initial recording, the dura was reflected, and the chamber was filled with silicone oil and sealed with a glass cover plate to stabilize the cortex for optical imaging.

Following optical imaging, the chamber was removed and more extensive electrode recordings were made. The cortex was covered with a layer of agarose (3\% in physiological saline) to reduce pulsations from respiratory motions. Electrolytic lesions were made to allow us to reconstruct the positions of the recording sites with respect to the subsequent $\mathrm{CO}$ histology (see Cytochrome oxidase histology and reconstruction).

We performed two to four recoveries on most animals. The number of experiments per craniotomy ranged from one to three. That is, we sometimes returned to the same recording site on a different experimental day to acquire additional data from a specific location. Animals were treated post-op with antibiotic (Tribrissen or Bactrin) and analgesics (Banamine and/or acetaminophen), and recovery surgeries were spaced at least $2 \mathrm{wk}$ apart with an average recovery of $1 \mathrm{mo}$. For terminal experiments, animals were overdosed with sodium thiopental and then perfused transcardially with a saline rinse followed by $4 \%$ paraformaldehyde.

\section{Cytochrome oxidase histology and reconstruction}

The exposed imaged tissue was lesioned at three or more locations at the end of an experimental session using a tungsten-recording electrode and a constant current injection (2-5 $\mu$ A for $2-5$ s, 200-900 $\mu \mathrm{m}$ deep). The locations of these lesions were marked on the vasculature map obtained during optical imaging. Post-mortem tissue was sectioned tangentially at 30-50 $\mu \mathrm{m}$ and reacted for $\mathrm{CO}$ activity using the procedure of Wong-Riley (1979). The tangential sections were then aligned with the optical images using the lesions as markers. Tissue was appropriately scaled between optical images and histological pictures using the magnification factor of the optical imaging lenses as well as the patterns of the lesions marked on the map compared to their location in the tissue. Finally, the registration of the ocular dominance columns from the optical maps with the $\mathrm{CO}$ blobs confirmed proper scaling and was sometimes used to make fine adjustments of $10 \%$ or less of the scale of the maps to the histology.

\section{Visual stimuli}

Stimuli for optical imaging consisted of equiluminant red/green and blue/yellow sinewave gratings of low spatial frequency ( 0.5 cycles/ $\mathrm{deg}$ ), achromatic luminance sinewave gratings of either high or low contrast (52 and 8\%), and of either high or low spatial frequency $(0.5$ or 1.5 cycles/deg), and finally $100 \%$ contrast square wave gratings. Color stimuli were modulated along either the red/green cardinal axis or the blue cone (tritanopic confusion) axis at maximal contrast. In some early cases, the red/green and blue/yellow stimuli were equiluminant but not calibrated to the cardinal axes. Instead, the red/green stimulus varied from pure red gun to pure green gun, and the blue/ yellow stimulus varied from pure blue gun to pure red + green gun. We will refer to these cases in the text as "noncardinal" stimuli. The stimulus gratings were presented at four different orientations: 0 (horizontal), 45,90 , and $135^{\circ}$. All gratings were drifted with a temporal frequency of 2 cycles/s.

All gratings had the same mean luminance $\left(37 \mathrm{~cd} / \mathrm{m}^{2}\right)$. Equiluminance was based on average human psychophysical responses (Smith et al. 1976) and was measured with a spectroradiometer (Photoresearch, Spectrascan, PR 703A). Differences between human and monkey luminance responses might lead to a residual luminance signal in our equiluminant chromatic stimuli. Because optical imaging analysis entails a differential measure, however, small residual responses due to luminance would have little effect on our visualization of color responses.

Electrode recordings were used to test responses of cells in dark patch-like features, detected during imaging, and the surrounding areas on optical maps. A hand-held projector was used to project stimuli on a white background of $\sim 1 \mathrm{~cd} / \mathrm{m}^{2}$ with room lights. Spots and bars of various wavelengths were presented using equal energy interference filters ranging from 450 to $630 \mathrm{~nm}$ in $30-\mathrm{nm}$ increments. Electrode recordings were made from the superficial cortical layers with glass-coated tungsten electrodes (Alan Ainsworth, London). Recordings included single-unit and multi-unit extracellular data.

Each cell or multi-unit cluster was tested first with a white bar for orientation selectivity, then with colored oriented bars, and finally with large and small colored spots. All cells recorded were classified according to the receptive field characteristics of ocular dominance, orientation selectivity, and color selectivity. Ocular dominance was ranked from 1 to 7 using the scale of Hubel and Wiesel (1962) where 1 refers to a purely contralateral response, 7 refers to a purely ipsilateral response, and 2-6 indicate varying degrees of binocularity. Orientation selectivity was classified as A through D using the scale of Livingstone and Hubel (1984), where A indicates sharp tuning, and $\mathrm{D}$ indicates unoriented responses. Color selectivity was documented according to the cells' responses to the different colored bars and spots and included notes on the occurrence of opponency and surround effects. The correspondence of these qualitative receptive-field 
properties with more objective measures is discussed in the following paper (Landisman and Ts'o 2002). Cells were categorized as color selective if they met the following criteria. First, the cells showed much stronger responses to certain wavelengths of light than to other wavelengths when stimulated with a spot that filled the receptive field center. For example, a cell was described as red-selective if its response was strongest to a stimulus of $630 \mathrm{~nm}$ (red) rather than to a stimulus of $510 \mathrm{~nm}$ (green), or $450 \mathrm{~nm}$ (blue). Second, the color cells had either a poor white response relative to the preferred wavelengths, or the white response was comparable to responses to the preferred wavelength (e.g., a cell that responded equivalently to a white stimulus and to a 630-nm stimulus but had little or no response to other wavelengths would be categorized as preferring red). In most cases color-selective cells were overtly color-opponent; such as a red/green cell that had a strong on response to a spot stimulus of $630 \mathrm{~nm}$ that decreased with decreasing wavelength and then changed sign to give an ofF response to green stimuli. Cells with peaks in the yellow range $(540$ or $570 \mathrm{~nm}$ ) were not considered color-selective unless they were color-opponent; that is, their responses changed sign at higher or lower wavelengths. Cells were characterized as broadband if they responded well to all wavelengths tested, often with slightly stronger responses in the middle wavelength ranges (around $570 \mathrm{~nm}$ ) than to the extremes (450 and $630 \mathrm{~nm}$ ).

When multiple single units were resolved at a given recording site, they generally had similar ocular dominance, orientation preference, and color selectivity. For this reason, we considered multiunit recordings to be an accurate representation of cell properties at a particular mapped location. Multiunit recording facilitated sampling of a greater number of regions.

\section{Optical imaging and analysis}

For optical imaging, a slow-scan charge-coupled device (CCD) camera (Photometrics) was focused on the surface of the exposed cortex. An image of the vasculature was obtained (for targeting electrode penetrations in relation to the functional imaging maps) by illuminating the brain with green $(540 \mathrm{~nm})$ light. The brain was illuminated with a red $(630 \mathrm{~nm})$ light source for data collection. This wavelength was selected to optimize the strength of the signal while minimizing blood vessel artifacts. The signal-to-noise ratio of the camera was measured to be approximately $1,400: 1$, and the spatial resolution of the CCD array was 192 by 144 pixels.

The light source was positioned to provide relatively uniform illumination of the cortical surface. Computer-controlled shutters were used to gate the visual stimuli to either eye. For sessions targeted just for the imaging of ocular dominance and orientation, the two eyes were kept in dark during the interstimulus interval (closed shutter protocol). Thus the shutter-gated presentation of the stimulus to an eye created a large luminance transient that is particularly effective for the imaging of ocular dominance columns. However, for the imaging of color responses, both eyes viewed a mean luminance background during the interstimulus interval, eliminating the initial luminance transient that might mask the weaker color response signals (open shutter protocol). On some occasions, ocular dominance was successfully mapped from images collected during the open shutter protocol, as illustrated in the example stimulus set in Fig. 1.

Ten individual frames of 300-ms duration were collected during a single stimulus presentation. A complete run or set generally consisted of 33 stimulus conditions (see Fig. 1 for a typical example of stimulus conditions used), 16 conditions for each eye plus a blank condition in which both eyes were open and a mean luminance field was displayed. Each run was repeated 16 times, with stimuli randomly interleaved. Optical imaging data were then analyzed on a Digital MicroVAX III computer or a Pentium PC. For analysis, the 16 repetitions and 10 frames for each condition were summed. Then each condition (for example, left-eye red/green horizontal) was normalized by dividing it by the average of all trials (including the no-stimulus conditions). This normalization eliminates a large common signal that can mask the stimulus-dependent signal for each condition.

Depending on the particular feature we wished to extract, the imaging data were summed across similar conditions. Pairs of sums were then subtracted to compute the differential response. For example, an ocular dominance map is generated by first adding together all images of left eye conditions (achromatic horizontal, vertical, $45^{\circ}$, and $135^{\circ}$ ), then adding together similar images from right eye stimulation, and finally subtracting the right-eye sum from the left-eye sum (see Fig. 1).

Contour plots of the outlines of features in the optical images, such as the one in Fig. $5 B$, were generated by creating a high-pass version of the image, to eliminate large-scale regional variability, and then by applying a low-pass filter to smooth edges of individual patches, and finally by using a thresholding algorithm in Photostyler (Aldus) or Photoshop (Adobe).

To avoid confusion, regions of dense staining for $\mathrm{CO}$ in histological sections of striate cortex will be referred to as $\mathrm{CO}$ blobs, and regions presumed to contain islands of color-selective neurons as revealed by optical imaging in vivo will be referred to as color patches.

\section{R E S U L T S}

Results are organized into three sections. The first section, Imaging of color patches, reports our findings associated with the red/green minus $52 \%$ luminance-contrast stimulation protocol, which was the most effective in locating color-selective regions. The second section, Comparison of color-imaging protocols, discusses several other protocols we tested for the imaging of color selectivity and the correlation of all tested protocols with electrophysiological recordings. The third section, Comparison of imaged color patches with ocular domi- conditions $\mathbf{0 - 7}$

conditions 8-15

conditions $16-23$

conditions 24-31

condition 32
Left Eye
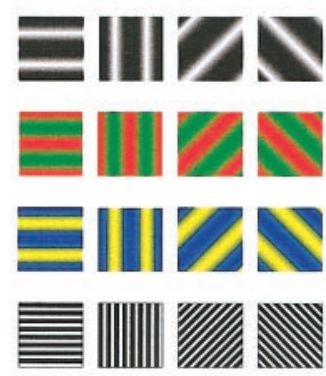

E.

\section{Right Eye}

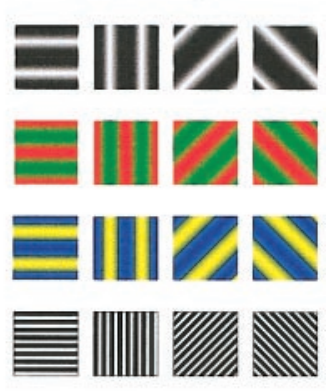

FIG. 1. The stimulus configuration delivered during an optical recording session. The trials are presented in random order, and multiples of each trial are collected. From these conditions, ocular dominance maps, color selectivity maps, and orientation maps can be generated. For example, color selectivity maps are generated by adding all the red-green conditions together (conditions 8-15) and subtracting out the sum of all the low spatial frequency achromatic luminance stimulus conditions (conditions $0-7$ ). The last condition (32) is a "blank," which can be subtracted from sets of conditions to eliminate baseline noise. 
nance, Cytochrome-oxidase staining and orientation, examines the relationship between the imaged color patches and other aspects of the functional architecture of V1, in particular the degree of overlap between color patches and $\mathrm{CO}$ blobs.

\section{Imaging of color patches}

In a typical experiment, first the vasculature was imaged (Fig. 2A) and then the protocol diagrammed in Fig. 1 was run, allowing for the differential imaging of several different modalities. Subtraction of all right-eye responses to luminance stimuli (Fig. 1, conditions 28-31; here 100\% contrast, 1.5 cycles/deg) from all left-eye responses (conditions 24-27) yielded ocular dominance maps (Fig. 2B). As in all maps, dark regions correspond to those that were more active during the "positive" term in the difference, here left-eye stimulation. Images of color responses (Fig. 2C) were obtained by subtracting responses to luminance gratings (conditions $0-7$ ) from the responses to red/green gratings at the same spatial frequency (0.5 cycles/deg; conditions 8-15). In this case, the red/green stimulus was equiluminant, but was not calibrated along the $\mathrm{red} /$ green cardinal axis. Nine of 10 cases generated similar maps with the same stimulus conditions. Despite the reliability of these maps, their features are generally harder to discern than those of ocular dominance maps for two reasons. First, the color patches are smaller and more irregular than ocular dominance columns. Second, the signal-to-noise ratio of color maps is roughly one-third that of ocular dominance maps.

We wished to exclude the possibility that this visualization of color patches might actually be due to sensitivity to different

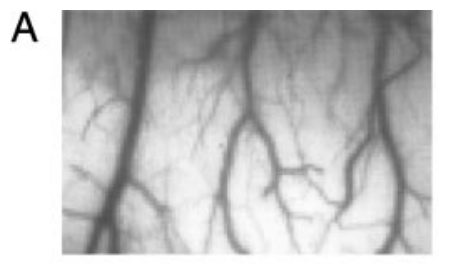

surface wl $570 \mathrm{~nm}$

B

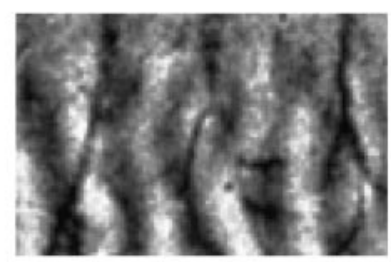

ocular dominance

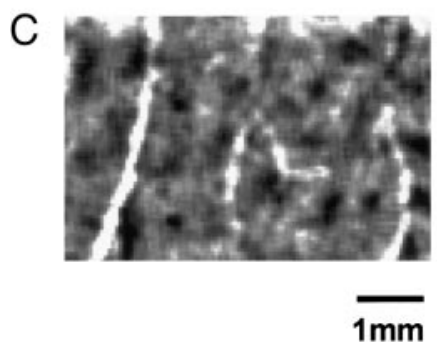

color - luminance

FIG. 2. Optical image of color selective patches in monkey striate cortex. $A$ : map of vasculature. Blood vessels appear as dark branches on the cortical surface. $B$ : map of ocular dominance from region seen in $A$. Dark columns correspond to the left eye and light columns to the right eye. $C$ : map of color-selective patches. Dark patches show regions more active during red/ green equiluminant stimulation than during broadband luminance stimulation. In this image, blood vessels appear as white lines and branches. luminance contrasts. For instance, our imaged patches might simply be regions with higher luminance contrast gain, as has been demonstrated for single neurons in the CO blobs (Edwards et al. 1995). We performed an independent control by comparing maps of color selectivity (red/green - luminance) to maps of low minus high luminance contrast (low luminance - high luminance). In four cases tested, no discernable features were seen in the low luminance - high luminance maps, although there were clear red/green - luminance maps (data not shown).

\section{Comparison of color imaging protocols}

To test the validity of the color maps obtained with different imaging protocols, we used electrophysiological recordings. Figure 3 shows one example of all five imaging protocols tested within one data set. In Fig. $3 A$, a map of red/green luminance is shown with and without electrophysiological recording sites. This panel shows a very good correspondence of color-selective penetrations (indicated by red and blue circles) with the dark patches in the optical image. By contrast, the other four map types seen in Fig. $3, B-E$, do not show good mapping features and do not appear to have any correspondence with the electrophysiology. That is, the slightly darker or lighter regions do not predict where the color penetrations fall compared to the broadband oriented penetrations. All the maps shown in Fig. 3 received identical image processing including high-pass filtering and contrast enhancement. Only Fig. 3D received additional darkening because the map was extremely light.

In addition to illustrating this individual data set shown in Fig. 3, we also compiled electrophysiological data from 139 penetrations ( $1-5$ cells recorded in each penetration, over 300 cells recorded in all from 7 different hemispheres in 7 animals) for the same five mapping protocols seen in Fig. 3 to determine the relative color selectivity of cells recorded in presumptive color patches (see Fig. 4; some penetrations were used to test more than one map type). Cells were categorized as "colorselective" qualitatively, as outlined in METHODS. The five different imaging protocols tested were 1) red/green - luminance, 2) red/green - blue/yellow, 3) red/green - blank, 4) blue/yellow - luminance, and 5) blue/yellow - blank. Each of these five protocols was imaged with color-axis modulated stimuli (7 experimental cases per protocol), and with equiluminant stimuli not calibrated to the cardinal axes but modulated between the pure gun values of the monitor (10 experimental cases per protocol). Not all of these 17 cases had electrophysiological data, so only 7 cases were used for Fig. 4. For the graphs in Fig. 4, the noncardinal axis modulation data were lumped with the cardinal axis modulation data since no significant differences were found between the two types. Of all the protocols, only the red/green - luminance cases consistently yielded images with detectable features (15/17 cases compared with 1-6/17 cases for the other 4 protocols tested shown in Fig. 4). The data in Fig. 4 were compiled only from the imaging cases that showed detectable features for each protocol.

Figure 4 shows examples of the correspondence of maps obtained from these optical images to electrophysiological recording. The data were analyzed by categorizing the electrode penetrations by their positions on the color map without 
A

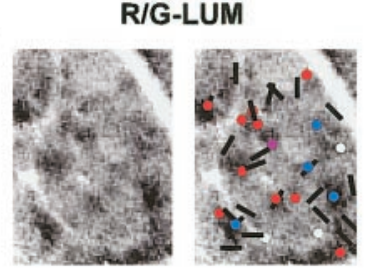

C

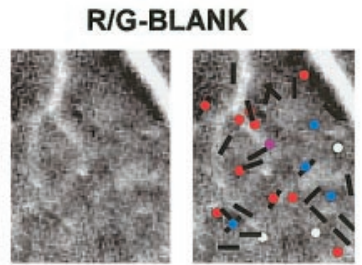

E
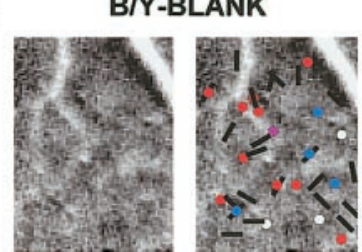

B

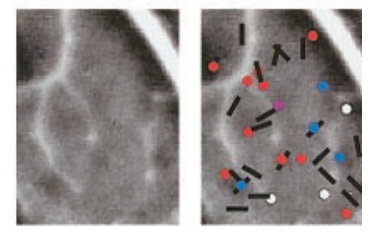

D

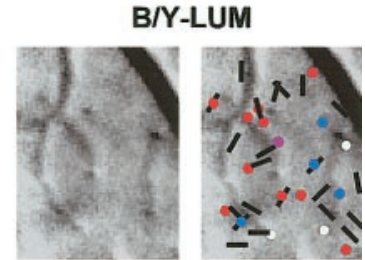

$\mathrm{F}$

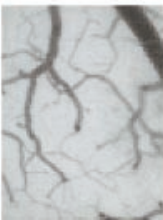

Vasculature

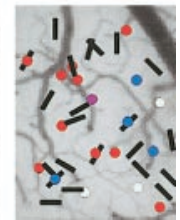

FIG. 3. Optical images of different mapping protocols with electrophysiological penetrations. Each map is shown twice: with and without electrophysiology icons. A: map of red/green minus luminance response (R/G - LUM). $B$ : map of red/green minus blue/yellow response (R/G - B/Y). $C$ : map of red/green minus blank response (R/G - BLANK). $D$ : map of blue/yellow minus luminance response (B/Y - LUM). E: map of blue/yellow minus blank response (B/Y - BLANK). $F$ : vasculature map from the same region shown in $A-E$. Red circles in all figures indicate penetrations with red/green selective unoriented cells. Blue circles in all figures indicate penetrations with blue/ yellow selective unoriented cells. White circles indicate broadband unoriented cells (type III). Black bars indicate broadband orientation-selective cells at the shown preferred orientation. Actual penetrations were located at the centers of the icon locations (see vasculature map). Penetrations with double icons had at least one of each cell type indicated. All maps were identically contrast enhanced.

referring to the documented electrophysiology of the penetrations. Penetrations were categorized as being within a dark region of a particular optical map, within a light region, or falling on the border. Border penetrations were defined as those less than $50 \mu \mathrm{m}$ from the light to dark transition of a patch and were not used in the histogram data.

Once the location was categorized, the basic color-selective properties for a given penetration were classified as broadband (BB), red/green opponent $(\mathrm{R} / \mathrm{G})$, or blue/yellow opponent $(\mathrm{B} / \mathrm{Y})$. Broadband penetrations contained cells that showed no color selectivity, whereas red/green and blue/yellow penetrations could also contain broadband cells. The main point of each histogram is to determine whether cell properties inside the dark regions could be distinguished from the properties in the lighter regions of a functional map. In each case, one stimulus condition type is subtracted from the other. The "positive" condition should dominate the cell properties of the dark regions, and the "negative" condition should dominate the light regions of the map. So, for example, in the case of R/G LUM (the top left histogram), the cells in the dark regions of the map should be more responsive to the red/green stimulus, and the cells in the light regions should be predominately broadband. This is, in fact, what is demonstrated in the histograms. The regions outside of the dark patches (indicated by light gray bars) are predominated by broadband cells with very

few color-selective cells. The regions inside the dark patches, on the other hand, have more color-selective cells than broadband cells. The differences between these two populations of cells in this case (inside or outside of the dark regions of the maps) are statistically significant $\left(P<<0.001\right.$ for a $\chi^{2}$ independence of variable test). Looking at the R/G - LUM case, it is very clear that the dark patches are dominated by red/greenselective penetrations and the light regions are dominated by broadband cells.

The only other map that shows a significant difference between the dark and light regions of the map is the R/G BLANK map, which also showed a predominance of red/green cells inside the dark patches and broadband cells outside of the patches. This was an unusual case, however, since it was the only R/G - BLANK map that had features. In general, we found that maps of individual conditions minus blank were featureless: only 1/17 cases yielded maps with features for the red/green minus blank case and $1 / 17$ for the blue/yellow minus blank case. This is likely to be due to a low signal/noise ratio. Because the overall activity of the cortex is greater during
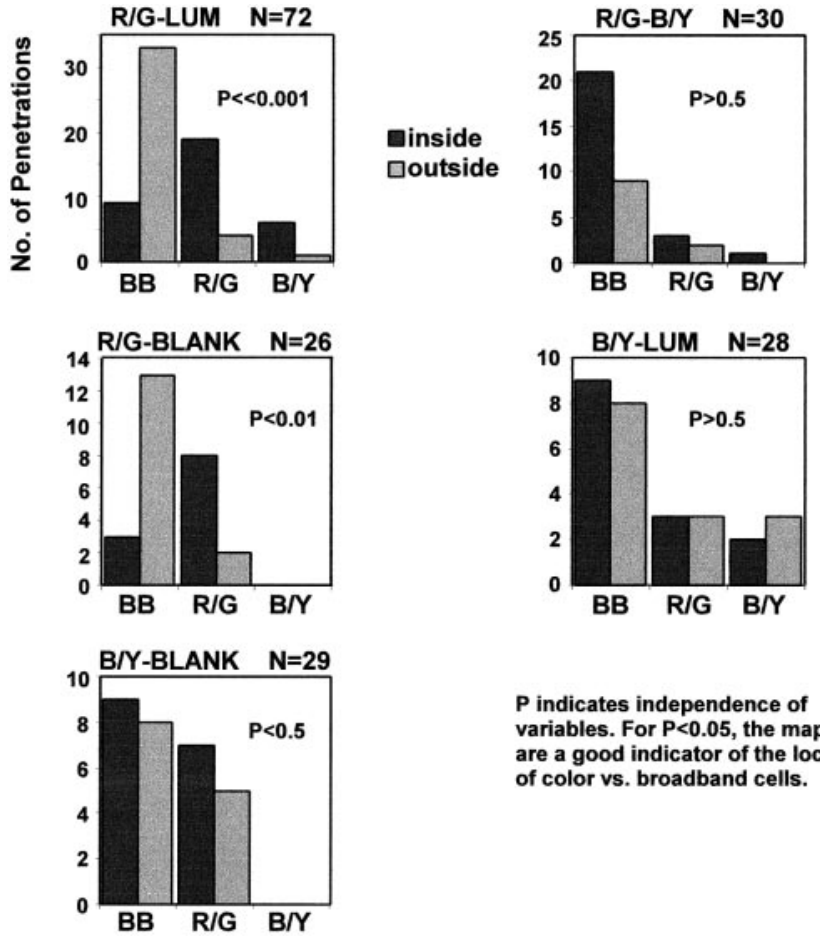

$P$ indicates independence of variables. For $P<0.05$, the maps are a good indicator of the location are a good indicator of the locati
of color vs. broadband cells.

FIG. 4. Histograms of the relationship between maps from different optical imaging protocols to electrophysiological recording. Each histogram indicates the number of penetrations predominated by broadband (BB), red/green opponent $(\mathrm{R} / \mathrm{G})$, and blue/yellow opponent $(\mathrm{B} / \mathrm{Y})$ cells inside dark regions of the maps vs. outside the dark regions. In each case, the dark regions should be an indicator of the locations of cells preferring the positive stimulus and the light regions (marked as outside) should indicate the locations of cells preferring the negative or subtracted stimulus. For example, in the top histograms, cells inside should respond better to the red/green stimulus and cells outside should respond better to the luminance stimulus. Statistical significance of the differences between the inside and outside populations were calculated using $\chi^{2}$ independence of variable tests. Because the populations of red/green and blue/yellow penetrations were too small to analyze as separate bins, they were pooled together into one bin of color-selective cells. As mentioned in the text, only the red/green - luminance cases consistently yielded images with detectable features. The histograms in this figure for the other map types were made using the few cases that actually yielded maps (thus the numbers are smaller for these cases). 
grating stimulation than during presentation of a gray screen (the blank condition), we would expect that the relatively weak differential mapping signal would be swamped by a larger stimulus-independent signal (see Frostig et al. 1990).

By looking at the remaining histograms in Fig. 4, one can see that the stimulus conditions showed no correlation to the color-selective properties of the electrophysiological penetrations. The $\mathrm{R} / \mathrm{G}-\mathrm{B} / \mathrm{Y}$ map does not show a predominance of $\mathrm{red} /$ green penetrations inside of the dark regions or a predominance of blue/yellow penetrations outside of the dark regions. Furthermore, the $\mathrm{R} / \mathrm{G}-\mathrm{B} / \mathrm{Y}$ maps were much less likely to even yield features (3/7 experiments for sessions using cardinal-axis modulating color stimuli, $3 / 10$ for sessions using noncardinal-axis modulating color stimuli). In the cases where comparison of $\mathrm{R} / \mathrm{G}-\mathrm{B} / \mathrm{Y}$ yielded a map with features, the map had patchy dark spots that appeared with a similar frequency, distribution, and size to the color patches normally seen in successful red/green minus luminance cases. Because these patches did not correspond strongly with color selectivity according to electrophysiological recording, we do not know what their origin was. The B/Y - LUM and B/Y - BLANK maps do not have significantly more blue/yellow selective penetrations inside the dark regions and are thus not good indicators of the location of blue/yellow selectivity or color selectivity in general.

In addition to the data presented in Fig. 4, more extensive electrophysiology of the red/green - luminance map is explored in the accompanying paper, where we show maps with electrophysiology and histograms from one large case including 74-80 recorded cells as well as histograms from 5 additional smaller cases documenting 149-173 cells (see Figs. 1, 3, and 5, companion paper, Landisman and Ts'o 2002).

In summary, we concluded that the most reliable color selectivity maps were generated by comparing high-contrast luminance stimulation to red/green stimulation, as confirmed by electrophysiological recordings and consistency of maps generated. This was true whether the equiluminant stimuli were modulated along the red/green cardinal axis or not. It is notable, however, that we were unable to image red/green versus blue/yellow opponent regions, although our electrode recordings did confirm that the two are segregated into different clusters (see the companion paper, Landisman and Ts'o 2002), as previously noted (Ts'o and Gilbert 1988). In that paper we show that less than $8 \%$ of the penetrations containing red/green or blue/yellow opponency contained cells of both of these types (7/91).

\section{Comparison of imaged color patches with $O D, C O$ staining, and orientation}

The color-selective images generated by red/green minus luminance stimulation were compared to OD maps, including centers of monocularity from the same region. OD maps were calculated by subtracting all right eye conditions from all left eye conditions obtained during stimulation with high spatial frequency, 100\% contrast luminance gratings (again, see Fig. 1, conditions 24-31, and Fig. 2B). These OD maps were then encoded in pseudocolor representation designed to highlight the regions of highest monocularity, which have been shown to correspond to CO blobs (Ts'o et al. 1990). In the pseudocolor maps (as seen in Fig. 5A), red codes for left eye columns, green for right eye columns, and blue for regions within each column showing the highest degree of monocularity. The map of color patches in Fig. 5C was created by comparing conditions for the left eye only-that is, left-eye red/green conditions minus left-eye luminance conditions.

Figure $5 B$ examines the overlap of the monocular zones of Fig. $5 A$ with the color-selective zones of Fig. $5 C$. In Fig. 5B, the patches in the image of color selectivity overlap the centers of monocularity in the OD image. When comparing the map in Fig. $5 C$ to the map of the most monocular regions in Fig. $5 A$, the color patches overlap only left eye monocular regions (see Fig. $5 B$ ). Consequently, the overlap of the color patches in Fig. $5 C$ with left eye regions of highest monocularity in $5 A$ indi-
A

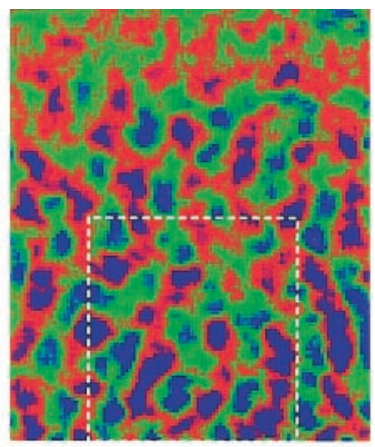

Ocular Dominance Map
B

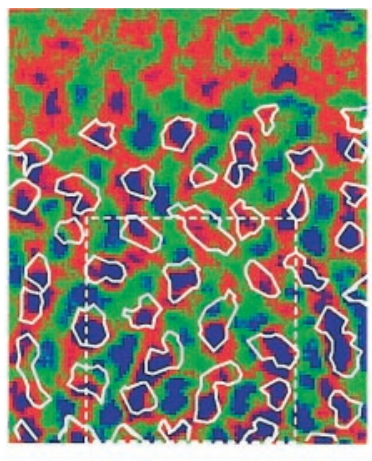

Overlay of Maps
C

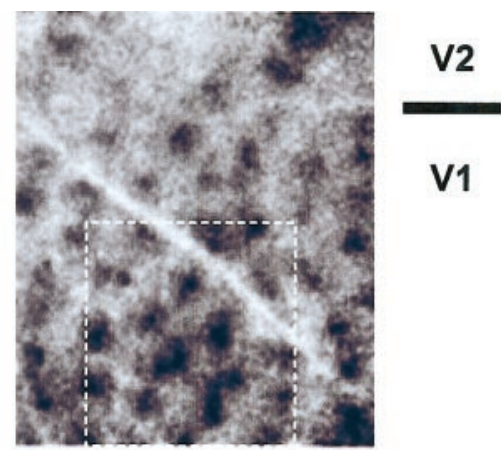

Monocular Color Map

$1 \mathrm{~mm}$

FIG. 5. Comparison of optical imaging of ocular dominance centers of monocularity to optical imaging of monocular color selectivity. A: a map in pseudocolor of ocular dominance. Left-eye columns are coded red, right-eye columns are coded green, and regions most responsive to only one eye are coded in blue and appear as patches along the centers of the red and green ocular dominance columns. $B$ : optical map from $A$ with a contour overlay from $C$. $C$ : optical map of color selectivity for the left eye, calculated by subtracting left-eye luminance conditions from left-eye red/green conditions. Note that virtually all the blue patches within red columns (left-eye monocularity centers) are overlapped by the color selective islands seen in $C$. Dotted outlines on all maps indicate borders of the optical maps shown in Fig. 6. Solid line to the right indicates the location of the V1/V2 border on all maps. 
cates the overlap of color patches with CO blobs. When a contour map of these color patches is superimposed on the monocular blob map (Fig. 5B), one can see that all of the left-eye monocularity regions (blue spots within red columns) are covered by the color patches. Thus there should also be a good correspondence of imaged color patches with $\mathrm{CO}$ blobs.

Despite the existence of many broadband cells within blobs (Lennie et al. 1990; Livingstone and Hubel 1984; Ts'o and Gilbert 1988), the regions of highest monocularity (and thus CO blobs) overlap with the optical map of color selectivity. These imaging results indicate that the blobs are regions with higher concentrations of color-selective cells even though electrophysiological recordings have indicated that many broadband cells lie within the $\mathrm{CO}$ blobs.

As noted above, comparison of the blue/yellow stimulus conditions to either the red/green conditions or to the achromatic conditions did not reveal blue/yellow selectivity. Nevertheless, both red/green and blue/yellow-selective color patches (as determined by electrophysiological recordings) appear in maps generated by subtracting achromatic conditions from $\mathrm{red} /$ green conditions. About $62.6 \%$ of penetrations within dark patches of red/green minus luminance maps contained exclusively red/green-selective cells (57/91), and $29.7 \%$ contained exclusively blue/yellow-selective cells (27/91). This is despite the fact that only $7.7 \%$ of penetrations containing red/green or blue/yellow opponent cells contained both classes of opponency (7/91; all penetrations had 2-6 cells each) (see companion paper, Landisman and Ts'o 2002). One possible explanation for the appearance of both types of color opponency could be that variations in the $\mathrm{L}$ (red) and $\mathrm{M}$ (green) cone balance could cause blue/yellow cells to be more responsive to the $\mathrm{red} /$ green gratings than to the luminance gratings. Yellow is created from $\mathrm{L}+\mathrm{M}$ cone inputs, but some blue/yellow cells have almost exclusively $\mathrm{L}$ or $\mathrm{M}$ inputs opposing their S-cone input. Furthermore, many color cells (particularly modified type II cells) (Ts'o and Gilbert 1988) have no response to luminance-modulated stimuli or are suppressed by them.

The appearance of the distribution of color patches and the regions of highest monocularity are similar. But upon closer inspection, it becomes clear that the color patches, although well correlated, are not identical to the monocularity centers. In Fig. $6 E$, an enlarged portion of the map for regions of greatest monocularity, seen in Fig. 5A, is compared to a map of color selectivity for both eyes from the same region of cortex (Fig. $6 B$ ). The color map in Figs. $5 C$ and $6 A$ (and 6C) shows color selectivity for only left-eye conditions. Figure $6, B$ and $D$, shows a color selectivity map in which conditions for both eyes have been added. In Fig. $6, E$ and $F$, the color maps are shown superimposed on maps of OD. Several color patches in the both eye map cross from one OD column to an adjacent column, and they overlap two neighboring regions of highest monocularity (Fig. $6 F$ ). Therefore the color patches are likely to be a superset of the most monocular regions, since they also cover binocular regions in between two neighboring OD columns. The fact that most or all regions of highest monocularity, and thus $\mathrm{CO}$ blobs, are encompassed by color patches might explain how limited sampling could give the impression that $\mathrm{CO}$ blob and color-selective cell distributions are completely identical.

Figure 7 shows an example of a comparison of $\mathrm{CO}$ blobs to the corresponding tissue imaged for color patches (electrolytic
Left Eye Color Map

A

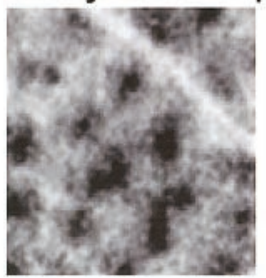

C

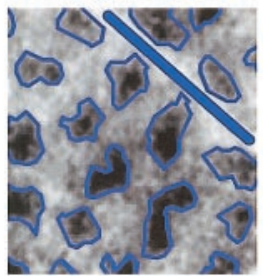

E

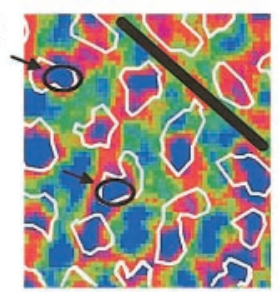

G

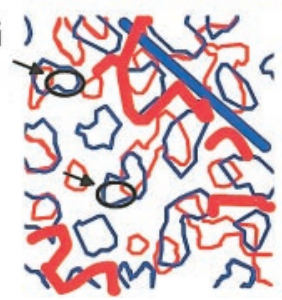

Both-Eye Color Map

B

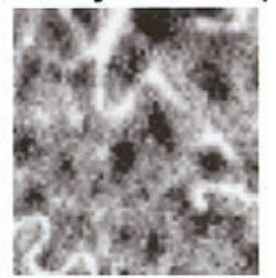

D

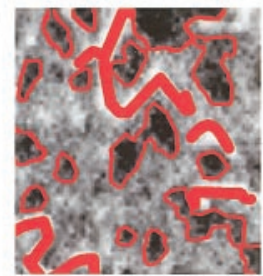

F

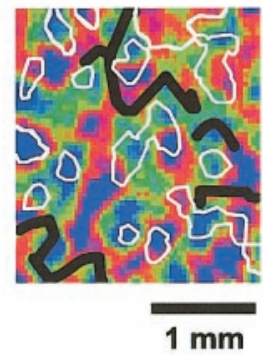

OD Map with outlines from color maps

Overlap of contours for left-eye color selectivity (blue) and both-eye color selectivity (red)

FIG. 6. Comparison of optical imaging of binocular color selectivity to maps of ocular dominance centers of monocularity. All panels are from a magnified region of the area shown in Fig. 5 (see dotted line outlines in Fig. 5). A: optical map of color selectivity for the left eye only (L eye R/G - L eye Lum). Color patches appear as dark islands. $B$ : map of color selectivity for both eyes: (L eye R/G $+\mathrm{R}$ eye R/G) - (L eye Lum + R eye Lum). Dark regions are more responsive to the $\mathrm{red} /$ green stimulus for either eye than to the high contrast luminance stimulus. This color selectivity map was generated using the same stimulus condition configuration as in Fig. $3 A$. $C$ : same map as in $A$ with blue contours drawn around the darkest regions and a blue line marking a blood vessel artifact. $D$ : same map as in $B$ with red contours drawn around the darkest regions and red lines marking blood vessel artifacts. $E$ : ocular dominance map coded in pseudocolor as in Fig. 4 (left eye columns are red, right eye columns are green, monocular regions are blue). White contours correspond to the left-eye color map (same contours as in $C$ ), and the black line is the same blood vessel artifact in $C$. F : same ocular dominance map as in $E$ with white contours from the both-eye color map. These are the same contours seen in $D$ including the blood vessel artifacts now coded in black. $G$ : overlay of the 2 contour drawings from left-eye color patches and both-eye color patches. Note that occasionally the both-eye patches appear smaller than the left-eye patches. This is due to scaling issues since purely monocular regions will respond to half as many conditions as binocular regions and might not appear dark. Thus some of the regions that appeared in the left-eye only map do not appear in the both-eye color map. Two examples of this are marked in this panel and in $E$ with arrows and black ellipses showing purely monocular (for the left eye) regions that appeared in the left-eye color map but were drowned out in the both-eye color map. The monocular nature of these encircled regions is confirmed by their blue appearance in the ocular dominance map of $E$.

lesions were used to align optical maps to CO histology, as mentioned in METHODS). In this example, one can see that the borders of the $\mathrm{CO}$ blobs and the color patches are fairly different, despite significant overlap. Two white arrows mark color patches (in $D$ and $E$ ) that spill over the corresponding CO blob borders. The top marked color patch overlaps a pair of $\mathrm{CO}$ 

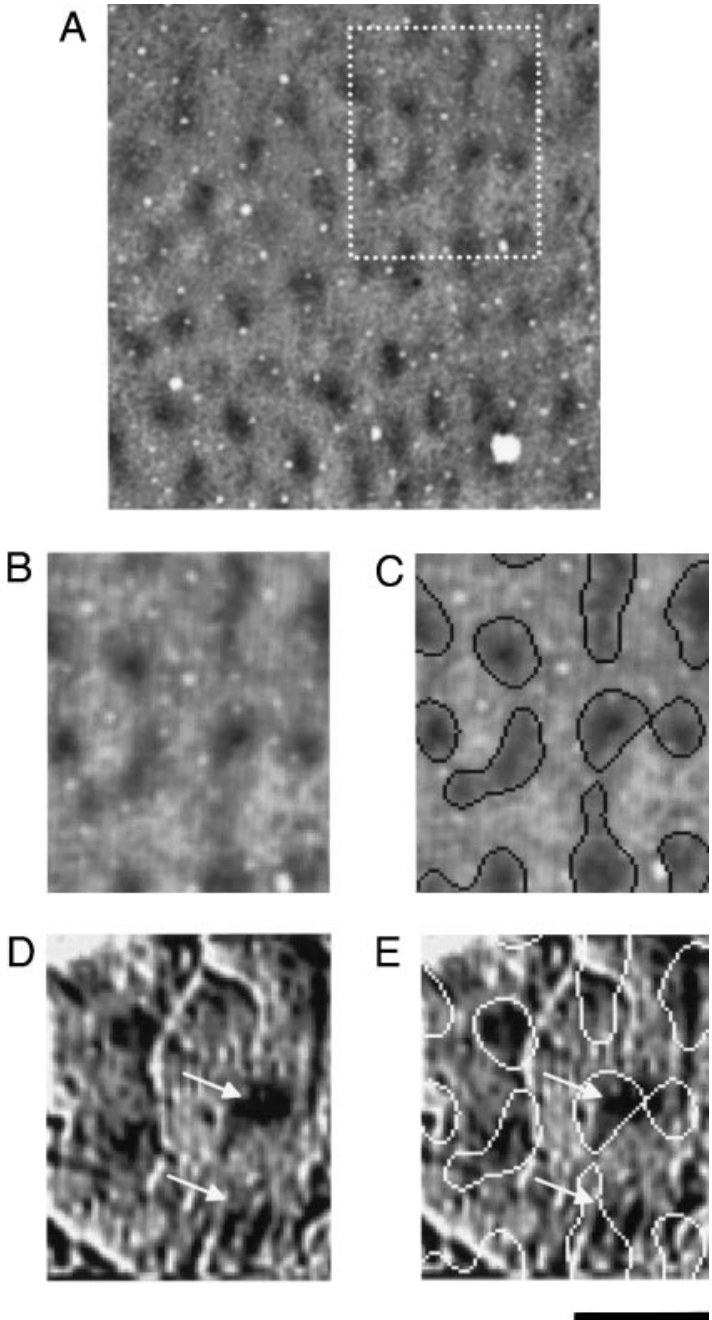

$1 \mathrm{~mm}$

FIG. 7. Comparison of cytochrome oxidase (CO) staining to imaging of color selectivity. A: tangential section stained for cytochrome oxidase. Dotted outline indicates the region to be compared to optical image in $D$ and $E$. $B$ : magnified view of the region outlined in $A$ showing staining for cytochrome oxidase. $C$ : Same section as $B$ with contours drawn around darkest regions. $D$ : optical image of color selectivity from the same region, scaled and aligned to the CO section. $E$ : same image as $D$ with contours from $C$ superimposed. The color patch overlapping the pair of blobs in the middle right (marked by the top arrow) appears to land directly in the middle of these 2 blobs without fully covering either blob. This is probably due to the scaling issue noted in the legend to Fig. 6; that is, purely monocular color selective regions are clipped compared to binocular color-selective regions that respond to red/green stimulation of both eyes as opposed to just one. The white arrows in $D$ and $E$ mark color patches that extend beyond a single $\mathrm{CO}$ blob. The top patch overlaps two blobs. The bottom patch, despite being larger than one blob, does not extend to a pair of blobs.

blobs, and the bottom color patch covers only one $\mathrm{CO}$ blob but extends well beyond its borders.

Using combinations of $\mathrm{CO}$ staining with maps of OD and color selectivity, we found examples of $\mathrm{CO}$ blobs paired across ocular dominance columns by a single color patch when compared with the optical image for both-eye color selectivity. Approximately $46 \%$ of color patches generated by these maps overlap with pairs of blobs (28/61 color patches from 4 cases), half along OD columns (14/61) and half across OD columns $(14 / 61)$. We also saw cases of single CO blobs that corresponded to a single imaged color patches. Thirty-six percent of color patches (22/61) were close to round in shape and covered only one blob each. Of these, 45\% (10/22) were large enough to cross OD borders. Eighteen percent of color patches (11/61) were irregular in shape. Overall, 56\% of color patches (34/61) crossed OD borders (this includes the 14/61 that encompassed pairs of blobs across OD columns as well as any color patches large enough to cross the borders without reaching the next neighboring blob). These observations suggest that the color patches seen in optical images may correspond to single $\mathrm{CO}$ blobs, or to combinations of two or more $\mathrm{CO}$ blobs lying across or along OD columns.

Despite the irregularity of the shape of color patches, there is still a good correlation of the location of color patches to the location of $\mathrm{CO}$ blobs. A calculation of the average distance from $\mathrm{CO}$ blobs centers to other $\mathrm{CO}$ blobs centers was similar to the average distance from color patch centers to color patch centers (0.82 vs. $0.68 \mathrm{~mm}$, respectively). Furthermore, the distance from color patch centers to $\mathrm{CO}$ blob centers is bimodal: the smaller average distance $(0.2 \mathrm{~mm}$, range $=0.05-$ $0.3 \mathrm{~mm}$ ) indicates that the distance is significantly smaller than the distance between pairs of CO blobs or color patches; the larger number $(0.92 \mathrm{~mm}$, range $=0.7-1.3 \mathrm{~mm})$ represents the distance to the next neighboring structure. These numbers were calculated based on the location of nearest neighbors.

Since we have determined that the relationship between CO blobs and color selectivity is not a perfect alignment, we felt that a comparison of other functional properties would help our understanding of how functional modules are laid out in striate cortex. The layout of orientation selectivity has already been compared with the ocular dominance columns (Blasdel 1992a) and CO blobs (Bartfeld and Grinvald 1992), but it has not yet been compared with the layout of color-selective patches. We were able to make these comparisons since our data set includes appropriate stimulus conditions to construct orientation maps as well as the color and OD maps from the same case. The orientation angle maps were generated by comparing different angle conditions using high-contrast grating stimuli (Fig. 1, conditions 24-31). These data were collected using a closed shutter protocol for the interstimulus interval (see МЕТнODS). Figure 8 shows an example of an orientation map compared with a map for OD, coded to enhance the most monocular regions, and a map for color selectivity. Figure $8 \mathrm{~A}$ illustrates a typical color map calculated by adding conditions for both eyes. $B$ shows the orientation selectivity map for the same region where orientation selectivity is color coded so that regions preferring the $90^{\circ}$ stimulus are yellow; $45^{\circ}$, red; $0^{\circ}$ (horizontal), blue; and $135^{\circ}$, green. Within the pattern of orientation selectivity, pinwheels can be seen where there is a full representation of all orientations rotate around a single point (Bonhoeffer and Grinvald 1991). The location of the pinwheel centers are then compared with the location of the color patches and OD columns and are marked with dots. As noted by several groups (Bartfeld and Grinvald 1992; Blasdel 1992b) and confirmed in our results, there is a tendency for lines of iso-orientation to cross OD columns at right angles, but the pinwheel centers do not necessarily fall on the CO blobs. Furthermore, we find no straightforward relationship of imaged color-selective patches to iso-orientation regions or pinwheel centers. We found that only $22 \%$ of pinwheel centers fall inside color patches (12/54 from 2 cases). This is consistent with the finding of Livingstone and Hubel (1984) that the interruption 

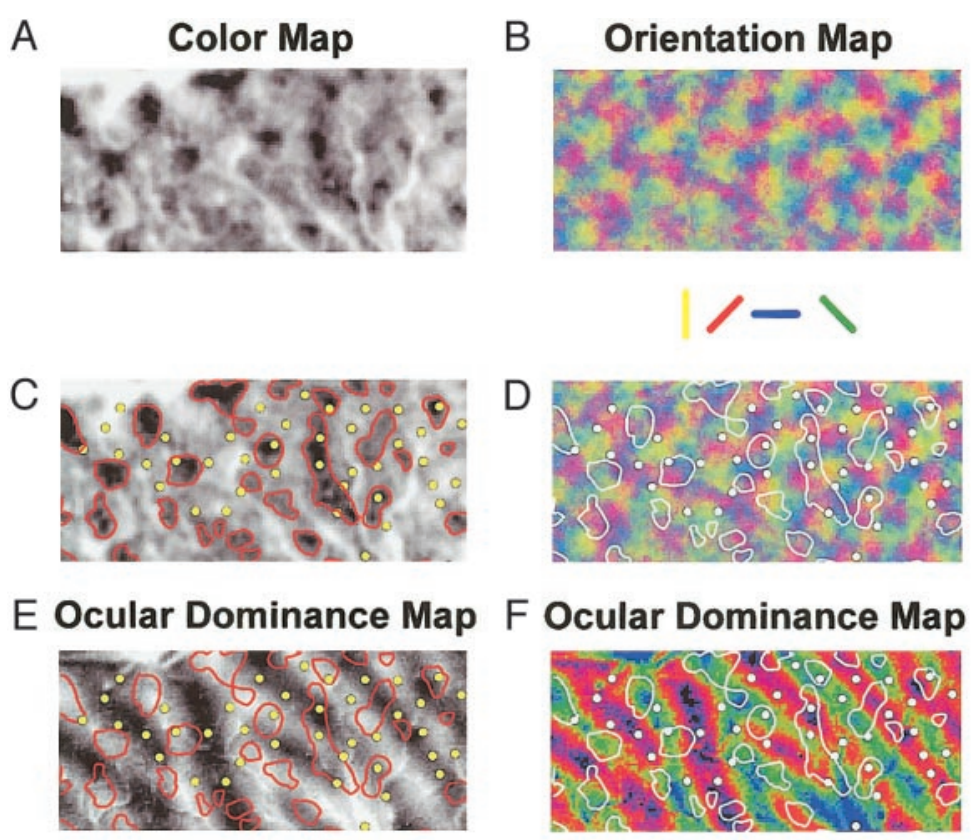

FIG. 8. Comparison of functional structures in V1. A: color selectivity map. $B$ : orientation map; yellow codes vertical, red codes $45^{\circ}$, blue codes horizontal, and green codes $135^{\circ}$. White and yellow dots mark pinwheel centers and black outlines show regions of color selectivity from $A$. $C$ : same color selectivity map shown in $A$ with red outlines showing the location of color patches and yellow dots showing the location of pinwheel centers from the orientation map in $B$. D: same orientation map shown in $B$ with the pinwheel centers marked by white dots and the location of color patches from the color map superimposed. $E$ : ocular dominance map with the location of pinwheel centers marked by yellow dots and the color patches outlined in black. $F$ : ocular dominance map in pseudocolor where left eye columns are red, right eye columns are green, and regions of strongest monocularity are blue. White dots show pinwheel centers from $D$, and black outlines show color-selective regions from $C$.

\section{$1 \overline{\mathrm{mm}}$}

of orientation progression by unoriented blob cells do not correspond to breaks or sudden changes in orientation, but that the orientation progression continues along a steady course before and after the interruption. Although most CO blobs (which lie along the centers of OD columns) are encompassed by color patches, individual color patches are not contained within OD columns. That is, the OD columns are strongly coupled to the $\mathrm{CO}$ blob layout, and the color patches are loosely in register with the CO blobs, but the OD columns and color patches have a variable relationship since the color patches sometimes cross columns and sometimes do not. The modules for orientation, color selectivity, and OD thus appear to comprise separate and largely independent representations.

\section{I S C U S S I O N}

Our data demonstrate that color selectivity in macaque V1 can be revealed using optical imaging. We called the colorselective structures seen in optical imaging "color patches" and compared them with other functional and anatomical features in V1. The color patches seen in our images encompass most $\mathrm{CO}$ blobs but do not correspond perfectly to $\mathrm{CO}$ staining. Instead, the imaged patches are often bigger than individual blobs and can contain pairs of blobs. Unlike the $\mathrm{CO}$ blobs, color patches are not confined to the centers of OD columns. In fact, many color patches cross OD columns and contain binocular color-selective cells. For this reason, the relationship of the color patches to OD columns is not as simple as previously suggested. Furthermore, there is no apparent relationship between the color patches and orientation pinwheel centers.

\section{Imaging of color-selective regions}

One interesting result that emerged from the optical imaging experiments is that all the color-selective regions were more responsive to $\mathrm{red} / \mathrm{green}$ equiluminant stimulation than to broadband stimulation. Perhaps a large contribution from type II (center color-opponent) and modified type II cells (cells that have color-opponent centers and broadband suppressive surrounds) (see Ts'o and Gilbert 1988) is responsible for this finding in our color maps. These cells have a strong coloropponent response but are suppressed by white light. This would imply that even blue/yellow cells of these two types would be less suppressed by a red/green stimulus than by an achromatic stimulus. In an optical image, this would be equivalent to being more responsive to the red/green stimulus than to the achromatic stimulus. Thus blue/yellow-selective regions would also be activated (and appear as dark patches) in a map of red/green minus achromatic stimulation.

We attempted to confirm the previously reported segregation of red/green and blue/yellow processing among the $\mathrm{CO}$ blobs of VI (Ts'o and Gilbert 1988) by using optical imaging in order to obtain a better understanding of the layout of each type of color module. The stimulus protocols we used in these experiments, however, were not sufficient to reveal this segregation. Simple subtraction of an achromatic stimulus activity from a blue/yellow stimulus activity does not reveal patterns of blue/ yellow selectivity as might be expected. Nor does subtraction of red/green stimulus activity from blue/yellow stimulus activity. Our electrophysiological findings, however, did confirm the segregation of red/green cells from blue/yellow cells within individual blobs as first observed by Ts'o and Gilbert (1988).

The inability to image red/green versus blue/yellow segregation could simply be a matter of not finding the optimal stimuli. One obvious problem could be improper scaling of two compared signals. To obtain a good optical image, the two relevant signals being compared must be of a similar magnitude. In the case of OD, this is fairly simple since there is a roughly equal representation of responses for each eye, both in terms of cortical territory and intensity of response. This is 
probably not the case for blue/yellow versus red/green or luminance since it is known that the representation for blue/ yellow is much sparser in V1 than for red/green or luminance (Ts'o and Gilbert 1988). For this reason, other stimulus sets should be tested to optimize the optical imaging of blue/yellow versus red/green selectivity. For instance, preliminary optical imaging data have suggested that a pattern of blue/yellow sensitivity is revealed with gratings modulated in the positive S-cone direction (Ts'o et al. 1998).

\section{Color imaging protocols}

Confirmation of color imaging by electrophysiological recordings revealed that only one mapping protocol accurately indicates the location of color patches. The equiluminant red/ green minus high-contrast luminance stimulus protocol was found to reliably generate maps with dark patches that were consistent with the electrophysiological properties of recorded cells. Protocols such as red/green minus blue/yellow, red/green minus blank, blue/yellow minus luminance, and blue/yellow minus blank did not adequately correspond to electrophysiological characterization of individual cells nor reliably generate maps.

\section{Comparison of maps: color patch imaging borders and $\mathrm{CO}$ borders}

In comparing the borders of color patches to regions of highest monocularity, monocular color patches, and $\mathrm{CO}$ blobs (see Figs. 5 and 6), we found that none of the features shared exact borders. When considering comparisons of borders, however, it should be noted that their determination must be subjective when comparing optical images to histological sections since the two cannot have equivalent thresholds. Even when comparing borders within images, one must assume that the signal sizes are similar for the different map types. Thus we were forced to draw qualitative conclusions of which structures were most similar to each other. Several explanations can account for the discrepancy of borders. First, although the images are scaled to best match, there is a certain amount of warping in histological tissue that could alter the relationship of the borders of the two structures. For instance, during optical imaging, the cortex is not nearly as flat as it is in the histology. Furthermore, due to the softness of the tissue, we have found that imaging identical structures on a region of cortex on different occasions can yield slightly different borders due to shifting of the less-than-rigid tissue, as best indicated by shifts in the vasculature.

Optical imaging is an indirect measure of neural activity that is derived from blood flow and blood oxygenation (Frostig et al. 1990). Therefore there may be a discrepancy between the imaged boundaries and the histologically defined borders of blobs. When we tested the borders of the imaged color patches with electrophysiology, we found a good correspondence within the limits of our electrode recording resolution (about $50 \mu \mathrm{m})$. Therefore we conclude that most color cells do fall within the $\mathrm{CO}$ blobs, as there is good overlap of imaged color patches with the $\mathrm{CO}$ blobs, but the borders of the two (as well as the average size of the structures) can be different.
Comparison of maps: the relationship of functional modules in striate cortex

Consistent with the electrophysiological findings of Livingstone and Hubel (1984) and Ts'o and Gilbert (1988), we found a strong correspondence between color patches and monocularity centers (which in turn correspond to the $\mathrm{CO}$ blobs). Blob cells do appear to be monocular and unoriented, if not always color-selective (also see Figs. 4 and 6, companion paper, Landisman and Ts'o 2002). This concept is supported by several findings: 1) most CO blobs lie along the centers of OD columns (Horton and Hubel 1981), 2) CO blobs show a high correspondence with centers of monocularity seen in optical imaging in vivo (Ts'o et al. 1990), and 3) the discrete staining is interrupted in layers $4 \mathrm{~A}$ and $4 \mathrm{C}$, which are continuously dark-stained and which contain almost exclusively monocular and unoriented cells (Blasdel and Fitzpatrick 1984).

The larger size of color patches compared with CO blobs (or the tendency of pairs of blobs to fall within individual color patches) may explain why Lennie et al. (1990) found color cells outside the CO blobs. Furthermore, the existence of broadband unoriented cells (type III) in the $\mathrm{CO}$ blobs as well as color-selective oriented cells outside of the $\mathrm{CO}$ blobs could additionally contribute to these earlier conclusions (Ts'o and Gilbert 1988).

Our results provide further information on the interrelationships between the functional maps of color, orientation, and ocular dominance. The main conclusion is that relationships between maps are much less ordered than in the various versions of the classic "ice cube" model of primate striate cortex (Horton 1984; Hubel and Wiesel 1977; Livingstone and Hubel 1984). It is interesting to consider the layout of CO blobs as a possible scaffolding upon which functional organization in V1 depends (Horton 1984). The OD columns are certainly in tight registration with the layout of the $\mathrm{CO}$ blobs since the blobs are centered on the middle of the OD columns. The striate organization of color processing is also largely dependent on the $\mathrm{CO}$ blobs since most of the color patches encompass one or more blobs. Indeed, one might think of color response properties as emanating from the $\mathrm{CO}$ blobs. The orientation map is most closely related to the OD columns since regions of iso-orientation tend to cross OD borders at right angles and since pinwheel centers tend to fall in the middle of OD columns (Bartfeld and Grinvald 1992; Blasdel 1992a,b).

These relationships of orientation and color to OD and $\mathrm{CO}$ blobs are not surprising, given the processing that must be accomplished in V1. Since V1 is retinotopic and its input is monocular, all properties must be represented for each eye. Thus, both orientation and color must be related to the layout of OD. At this cortical stage, however, there may not be a need to construct a precise relationship or interface between the cortical maps for color and orientation since these two maps are fairly segregated. That is, one would not expect a full retinotopic representation of all orientations along both cardinal color axes in V1. Evidence from studies in V2 (Burkhalter and Van Essen 1986; Roe and Ts'o 1995) and V4 (Ghose and Ts'o 1997) suggests that these subsequent visual areas have a greater role in the processing of the combination of color and orientation. Thus the representations for color and orientation may not require an interdependence and rigid registration with each other in their layout in V1. 


\section{Summary}

From our results, we conclude that color cells in the superficial layers of macaque striate cortex are clustered together in patches that are largely coextensive with the CO blobs. Our finding that the color patches extend into "non-blob" cortical territory (as defined histologically) might help explain discrepancies in earlier electrophysiological literature on blob cell properties. The loose relationship that we observed between the color patches and the various other functional maps in striate cortex indicates some degree of independence of these representations rather than a precise registration with each other (Blasdel 1992a). Therefore these results do not support the notion of a strict "hyper-module" that encompasses all the functional representations at each point in visual space, such as depicted in the classic ice cube model.

We thank S. Zagorski, C. Lorusso, L. Hinderstein, and A. Meyer for technical assistance and J. Maunsell and E. Kaplan for help with organization and writing.

This work was supported by National Institutes of Health Grants GM07524-15 and EY-08240, Office of Naval Research Grant N00014-91-J-1865, the Whitaker Foundation, the McKnight Foundation, and Helen Hay Whitney Foundation support to C. E. Landisman.

Present address of D. Y. Ts'o: Neurosurgery/Physiology IHP4111, SUNY Health Science Center, 750 East Adams St., Syracuse, NY 13210.

\section{REFERENCES}

Allman J AND ZuCKeR S. Cytochrome oxidase and functional coding in primate striate cortex: a hypothesis. CSH Symposium in Quant Bio, 55: 979-982, 1990.

BARTFELD E AND GRINVALD A. Relationships between orientation-preference pinwheels, cytochrome oxidase blobs, and ocular-dominance columns in primate striate cortex. Proc Natl Acad Sci USA 89: 11905-11909, 1992.

BLASDEL GG. Topography of visual function as shown with voltage sensitive dyes. In: Sensory Systems in the Mammalian Brain, edited by Lund JS. New York: Oxford UP, 1989a, p. 242-268.

BLASDEL GG. Visualization of neuronal activity in monkey striate cortex. Annu Rev Physiol 51: 561-581, 1989b.

BLASDEL GG. Differential imaging of ocular dominance and orientation selectivity in monkey striate cortex. J Neurosci 12: 3117-3140, 1992a.

BLASDEL GG. Orientation selectivity, preference, and continuity in monkey striate cortex. J Neurosci 12: 3141-3163, 1992b.

Blasdel GG AND FitzPatrick D. Physiological organization of layer 4 in macaque striate cortex. J Neurosci 4: 880-895, 1984.

Blasdel GG and SAlama G. Voltage-sensitive dyes reveal a modular organization in the monkey striate cortex. Nature 321: 579-585, 1986.

BoNHOEFFER T AND GRINVALD A. Iso-orientation domains in cat visual cortex are arranged in pinwheel-like patterns. Nature 353: 429-431, 1991.

BOYNTON RM. A system of photometry and colorimetry based on cone excitations. Color Res Appl 11: 244-252, 1986.

Brattenburg V and BRAITEnburg C. Geometry of orientation columns in the visual cortex. Biol Cybern 33: 179-186, 1979.

Burkhalter A AND VAn Essen DC. Processing of color, form and disparity information in visual areas VP and V2 of ventral extrastriate cortex in the macaque monkey. J Neurosci 6: 2327-2351, 1986.

DAW NW. The psychology and physiology of colour vision. Trends Neurosci 7: 330-335, 1984

Dow BM. Functional classes of cells and their laminar distribution in monkey visual cortex. J Neurophysiol 37: 927-946, 1974.

Dow BM AND Gouras P. Color and spatial specificity of single units in rhesus monkey foveal striate cortex. J Neurophysiol 36: 79-100, 1973.

EdWARds DP, PURPURA KP, AND KAPLAN E. Contrast sensitivity and spatial frequency response of primate cortical neurons in and around the cytochrome oxidase blobs. Vision Res 35: 1501-1523, 1995.

Frostig RD, Lieke EE, Ts'o DY, AND Grinvald A. Cortical functional architecture and local coupling between neuronal activity and the microcirculation revealed by in vivo high-resolution optical imaging of intrinsic signals. Proc Natl Acad Sci USA 87: 6082-6086, 1990.
GHOSE GM AND Ts'o DY. Form processing modules in primate area V4. J Neurophysiol 77: 2191-2196, 1997.

Gouras P. Opponent-colour cells in different layers of foveal striate cortex. J Physiol (Lond) 238: 583-602, 1974.

Gouras P AND KRUGER J. Responses of cells in foveal visual cortex of the monkey to pure color contrast. J Neurophysiol 42: 850-860, 1979.

Gouras P AND ZRENNER E. Color vision: a review from a neurophysiological perspective. Prog Sensory Physiol 1: 139-179, 1981.

Grinvald A, Frostig RD, Lieke E, And Hildesheim R. Optical imaging of neuronal activity. Physiol Rev 68: 1285-1366, 1988.

HorTON JC. Cytochrome oxidase patches: a new cytoarchitectonic feature of monkey visual cortex. Philos Trans R Soc Lond B Biol Sci 304: 199-253, 1984.

HoRTON JC AND Hubel DH. Regular patchy distribution of cytochrome oxidase staining in primary visual cortex of macaque monkey. Nature 292: 762-764, 1981 .

Hubel DH AND Livingstone MS. Color and contrast sensitivity in the lateral geniculate body and primary visual cortex of the macaque monkey. $\mathrm{J} \mathrm{Neu}$ rosci 10: 2223-2237, 1990.

Hubel DH AND Wiesel TN. Receptive fields, binocular interaction and functional architecture in the cat's visual cortex. J Physiol (Lond) 160: 106-154, 1962.

HubEL DH AND WIESEL TN. Functional architecture of macaque monkey visual cortex (Ferrier Lecture). Proc R Soc Lond B Biol Sci 198: 1-59, 1977.

JACOBS GH. Visual capacities of the owl monkey (Aotus trivirgatus). I. Spectral sensitivity and colour vision. Vision Res 17: 811-820, 1977.

Krauskopf J, Williams DR, AND HeEley DW. Cardinal directions of color space. Vision Res 22: 1123-1131, 1982.

LACHICA EA, BECK PD, AND CASAGRANDE VA. Parallel pathways in macaque monkey striate cortex: anatomically defined columns in layer III. Proc Natl Acad Sci USA 89: 3566-3570, 1992.

LACHICA EA And CASAgRAnde VA. Direct W-like geniculate projections to the cytochrome oxidase (CO) blobs in primate visual cortex: axon morphology. J Comp Neurol 319: 141-158, 1992.

LANDISMAN CE, GRINVALD A, AND Ts'o DY. Optical imaging reveals preferential labeling of cytochrome oxidase-rich regions in response to color stimuli in areas V1 and V2 of Macaque monkey. Soc Neurosci Abstr 17: 431.6, 1991.

LANDISMAN CE AND Ts'o DY. Color processing in the cytochrome oxidaserich blobs and bridges of macaque striate cortex. Soc Neurosci Abstr 18: 256.3, 1992.

LANDISMAN CE AND Ts'O DY. Color processing in macaque striate cortex: electrophysiological properties. J Neurophysiol 87: 3138-3151, 2002.

LENNIE P. Recent developments in the physiology of color vision. Trends Neurosci 7: 243-248, 1984.

LENNIE P, KRAUSKOPF J, AND SClaR G. Chromatic mechanisms in striate cortex of macaque. J Neurosci 10: 649-669, 1990.

Leventhal AG, Thompson KG, Liu D, Zhou Y, and Ault SJ. Concomitant sensitivity to orientation, direction, and color of cells in layers 2,3 , and 4 of monkey striate cortex. J Neurosci 15: 1803-1818, 1995.

Lieke EE, Frostig RD, Arieli A, Ts'o DY, Hildesheim R, and Grinvald A. Optical imaging of cortical activity: real-time imaging using extrinsic dye-signals and high resolution imaging based on slow intrinsic-signals. Annu Rev Physiol 51: 543-559, 1989.

LivingSTONE MS AND HubEL DH. Anatomy and physiology of a color system in the primate visual cortex. J Neurosci 4: 309-356, 1984.

MACLEOD DIA AND BoynTON RM. Chromaticity diagram showing cone excitation by stimuli of equal luminance. J Opt Soc Am 69: 1183-1186, 1979.

MARTIN KAC. From enzymes to visual perception: a bridge too far? Trends Neurosci 11: 380-387, 1988.

RoE AW AND Ts'o DY. Visual topography in primate V2: multiple representation across functional stripes. J Neurosci 15: 3689-3715, 1995.

SCHILler PH AND COlBy CL. The responses of single cells in the lateral geniculate nucleus of the rhesus monkey to color and luminance contrast. Vision Res 23: 1631-1641, 1983.

SCHILlER PH, LOGOTHETIS NK, AND CHARLES ER. Roles of color-opponent and broad-band channels in vision. Visual Neurosci 5: 321-346, 1990.

SCHILler PH, Logothetis NK, AND Charles ER. Parallel pathways in the visual system: their role in perception at isoluminance. Neuropsychologia 29: 433-441, 1991.

Silverman MS, Grosof DH, De Valois RL, And Elfar SD. Spatial-frequency organization in primate striate cortex. Proc Natl Acad Sci USA 86: 711-715, 1989. 
SMITH VC, POKORNY J, AND STARR SJ. Variability of color mixture data-I. Interobserver variability in the unit coordinates. Vision Res 16: 1087-1094, 1976.

Thorell LG, DeValois RL, And Albrecht DG. Spatial mapping of monkey V1 cells with pure color and luminance stimuli. Vision Res 24: 751-769, 1984.

Tootell RBH, Silverman MS, Hamilton SL, De Valois RL, and Switkes E. Functional anatomy of macaque striate cortex. III. Color. J Neurosci 8: 1569-1593, 1988.

Ts'o DY. The functional organization and connectivity of color processing. In: Neural Mechanisms of Visual Perception, Chapter: Structure and Function of the Visual Cortex, edited by Lamb TD and Gilbert CD. Houston, TX: Gulf Publishing, 1989, p. 97-115.

Ts'O DY, BURKITT GR, AND LEE J. The progression of color processing in visual areas V1, V2, and V4 of the macaque monkey. Soc Neurosci Abstr 24: $788.1,1998$
Ts'o DY, Frostig RD, LIEKE EE, AND GRINVALD A. Functional organization of primate visual cortex revealed by high resolution optical imaging. Science 249: 417-420, 1990 .

Ts'o DY AND GILBERT CD. The organization of chromatic and spatial interactions in the primate striate cortex. J Neurosci 8: 1712-1727, 1988.

Wiesel TN AND HubEL DH. Spatial and chromatic interactions in the lateral geniculate body of the rhesus monkey. J Neurophysiol 29: 1115-1156, 1966.

WONG-RILEY M. Changes in the visual system of monocularly sutured or enucleated cats demonstrable with cytochrome oxidase histochemistry. Brain Res 171: 11-28, 1979.

ZEKI S. Colour coding in the cerebral cortex: the responses of wavelengthselective and colour-coded cells in monkey visual cortex to changes in wavelength composition. Neuroscience 9: 767-781, 1983.

Zheng D, LaMantia A-S, And Purves D. Specialized vascularization of the primate visual cortex. J Neurosci 11: 2622-2629, 1991. 